Ca

the $\gamma \delta$ T cells

become

activated by

the adipose

tissue stress

during early

weight gain controls and had increased fasting blood levels of glucose. After the long-term ketogenic diet, the number of macrophages had increased and the number of $\gamma \delta$ T cells had decreased.

"Our data raise the possibility that the $\gamma \delta$ T cells become activated by the adipose tissue stress during early weight gain and are important for maintaining homeostasis and metabolic health long term," say Dixit and first author Emily Goldberg. The researchers also showed that glucose homeostasis is impaired in mice lacking $\gamma \delta$ T cells, adding further evidence to the important role of these cells in metabolic health.

"Our results emphasize that fat breakdown to produce ketone bodies, at the organismal level, can occur simultaneously with increased fat storage," concludes Dixit. "How these two 'opposing' programmes are coordinated within a single animal is an important area of biology that requires further study."

Claire Greenhill

ORIGINAL ARTICLE Goldberg, E. L. et al. Ketogenesis activates metabolically protective $\gamma \delta$ T cells in visceral adipose tissue. Nat. Metab. 2 , 50-61 (2020)

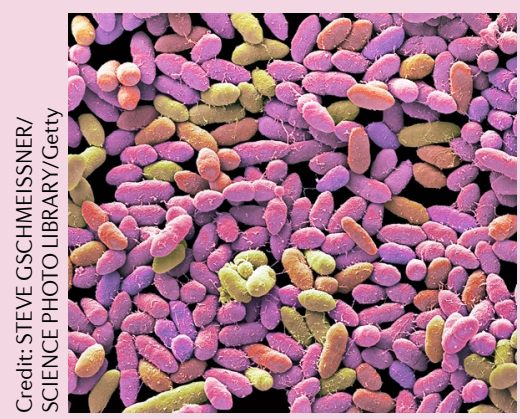

metabolism is currently unclear. "I think this is a critical question, which we hope to partially address with our international trials and potential future medication trial," adds Cree-Green.

The team are now enrolling participants for two clinical trials in which the authors will examine whether a nutritional intervention can change the composition of the microbiota and whether any changes also relate to changes in metabolic health.

Alan Morris

ORIGINAL ARTICLE Jobira, B. et al. Obese adolescents with PCOS have altered biodiversity and relative abundance in gastrointestinal microbiota.J. Clin. Endocrinol. Metab. https:// doi.org/10.1210/clinem/dgz263 (2020)

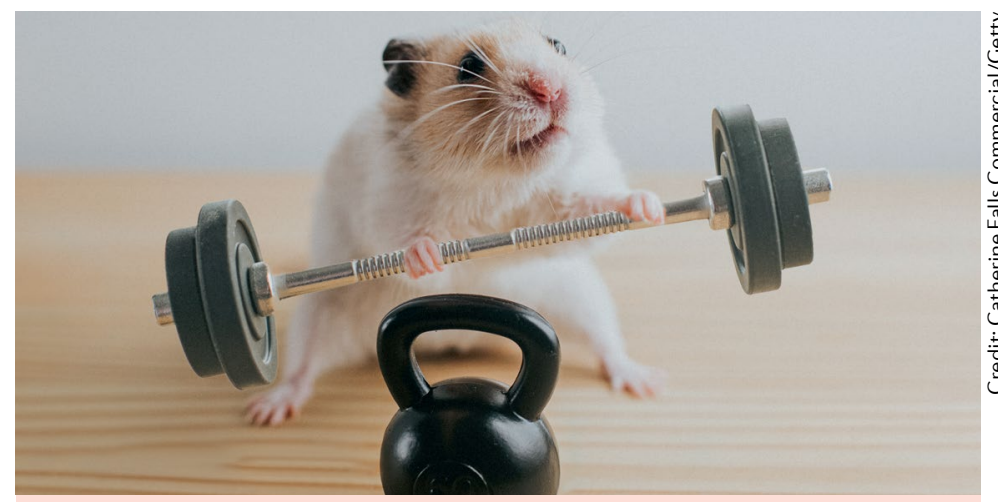

EXERCISE

\section{Role for toll-like receptor 9 in muscle AMPK activation}

Exercise induces autophagy in skeletal muscle and activation of the master regulator of energy homeostasis, AMP-activated protein kinase (AMPK). A study in Nature now reports a previously undescribed role for an innate immune toll-like receptor (TLR) in skeletal muscle metabolism during exercise.

"Our lab previously discovered that exercise induces autophagy (in skeletal muscle and other tissues) and that the autophagy protein, Beclin 1, is required for exercise-induced AMPK activation in skeletal muscle," explain corresponding authors Yang Liu and Beth Levine. "This finding raised the intriguing possibility that autophagy, or at least certain autophagy proteins, might function upstream of AMPK; however, the mechanism by which this effect might occur was unknown."

A proteomics screen in HeLa cells identified TLR7 and TLR9 as proteins that interacted with a functionally important region of Beclin 1. TLR9 senses mitochondrial DNA ( $m t D N A)$ and initiates an innate immune response. Of note, exogenous TLR9 ligands are known to activate AMPK in cardiomyocytes and neurons. "We hypothesized that TLR9 and its interaction with Beclin 1 might function in the regulation of exercise-induced AMPK activation in skeletal muscle," explain Liu and Levine.

Endogenous TLR9 expression is difficult to detect, so the researchers generated a knock-in mouse model expressing haemagglutinin (HA)-tagged TLR9. When these TLR9-HA mice were exercised on a treadmill, TLR9 interacted with both Beclin 1 and mtDNA in a time-dependent manner.

Next, a previously generated whole-body TLR9-knockout mouse (TLR9-KO) was used to show that TLR9 is crucial for exercise-induced AMPK activation in skeletal muscle. In addition, exercised TLR9-KO mice showed decreased GLUT4 translocation to the myocyte membrane and decreased glucose uptake by muscle.

Bone marrow transplantation experiments between wild-type and TLR9-KO mice were used to rule out the importance of haematopoietic cells for exercise-induced AMPK activation. Of note, ex vivo electrical stimulation of wild-type and TLR9-KO muscle also showed that TLR9-KO muscles had decreased AMPK activation and decreased glucose uptake.

Finally, experiments in muscle from wild-type and TLR9-KO mice revealed that the mechanism for TLR9-Beclin 1 induced muscle AMPK activation is through increased levels of a Beclin 1-UVRAG complex.

"Our study demonstrates unexpected crosstalk between an immune sensor, TLR9, and an autophagy protein, Beclin 1, in the control of energy metabolism in skeletal muscle during exercise," conclude Liu and Levine. "Our finding might present a more general paradigm whereby these two important cellular pathways intersect to maintain normal mammalian physiology."

Shimona Starling

ORIGINAL ARTICLE Liu, Y. et al. TLR9 and beclin 1 crosstalk regulates muscle AMPK activation in exercise. Nature https://doi.org/10.1038/s41586-020-1992-7 (2020) 